\title{
Social Work and the City: Urban Themes in 21st- Century Social Work
}

\section{Felipe Saravia-Cortés}

Doctor en Ciencias Sociales en Estudios Territoriales. Trabajador social Universidad del Bío-Bío, Concepción, Chile https:// orcid.org/0000-0003-3196-7831・fsaravia@ubiobio.cl

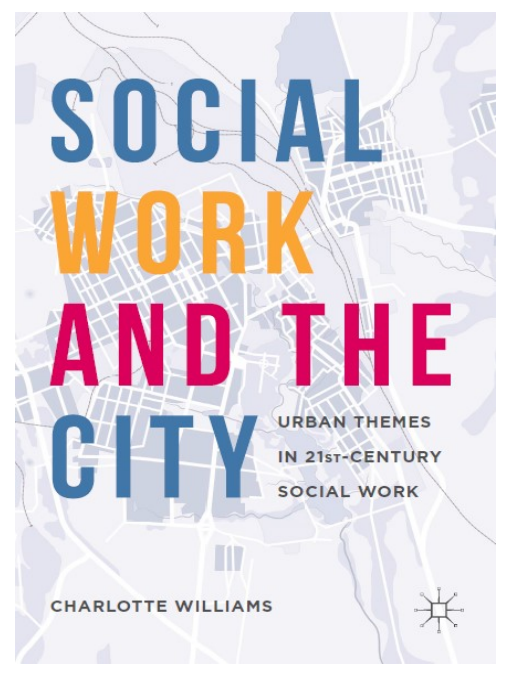

Williams, Charlotte (Ed.). 2016

Social Work and the City: Urban

Themes in 21st-Century Social Work.

Melbourne, Australia: Palgrave

Macmillan.

$\mathrm{XV}, 299 \mathrm{p}$.

ISBN: 978-1-137-51622-0

eBook ISBN: 978-1-137-51623-7

Trabajo Social ha desarrollado desde sus inicios en el contexto anglosajón, un vínculo profundo con la realidad urbana. En efecto, el movimiento de Settlements y el trabajo de precursoras como Jane Addams u Octavia Hill demuestra un interés permanente por cómo se entrecruzan las condiciones sociales de vida de las comunidades con las que se trabaja, así como las condiciones materiales de las ciudades y barrios en que estas desarrollan su vida cotidiana (Addams, 2013; Wohl, 1971). Sin embargo, como varios autores lo han constatado recientemente (Saravia, Urquieta y Ortega, 2020; Abreu, 2018; Williams, 2016; Orellana y Pánez, 2016; Zapf, 2009), durante las últimas décadas, en Trabajo Social han predominado perspectivas que no tienen en suficiente consideración la espacialidad de los fenómenos sociales que se atienden, y del quehacer de la intervención social.

Recibido: 08/03/2020 | Aprobado: 12/08/2020 |Publicado: 01/01/2021

c) (i) Esta obra está bajo una Licencia Creative Commons Atribución-NoComercial-CompartirIgual 4.0 Internacional.

\section{¿Cómo citar este artículo? / How to quote this article?}

Saravia-Cortés, F. (2021). Social Work and the City: Urban Themes in 21st-Century Social Work. Prospectiva. Revista de Trabajo Social e intervención social, (31), 457-462. doi: 10.25100/prts.v0i31.9044. 
Saravia-Cortés

Por esta razón, el trabajo de Charlotte Williams (2016) constituye un aporte importante ya que, partiendo de la constatación de que el mismo Trabajo Social es un producto de la vida en la ciudad, fundamenta la idea de que este debiese incorporar en la actualidad una perspectiva urbana, para enfrentar diversos ámbitos de su quehacer, tales como la lucha contra la pobreza, el abordaje de la diversidad demográfica, étnica y religiosa, y el trabajo con adultos mayores, con personas en situación de discapacidad, y con personas en situación de calle. Todos estos temas son abordados en capítulos específicos que configuran la segunda parte del libro, en los que autores invitados, especialistas en cada uno de estos tópicos, reflexionan acerca del vínculo entre la ciudad y el Trabajo Social o, en otras palabras, qué implica en términos concretos el uso de una perspectiva urbana en el tratamiento de cada uno de estos asuntos.

Ahora bien, desde mi punto de vista, el principal aporte del libro se encuentra en la primera parte, en donde la autora, luego de fundamentar la importancia de las ciudades en la vida contemporánea -no solo para explicar las causas espaciales de los fenómenos en relación con los que se desarrolla el quehacer del Trabajo Social, sino también para la identificación de oportunidades para el enfrentamiento de problemas sociales- delinea de forma somera algunas perspectivas teóricas que intentan explicar la ciudad desde disciplinas tales como los estudios urbanos, la geografía, la sociología, entre otras, haciendo referencia a autores clásicos -como Friedrich Engels, Emile Durkheim, Max Weber, Karl Marx y Georg Simmel- en cuyas obras es posible rastrear cierto pensamiento acerca de las condiciones de vida urbanas de su época en sus respectivas sociedades, y a autores más explícitamente vinculados a asuntos urbanos, como aquellos que conformaron la Escuela de sociología urbana de Chicago, y otros como Manuel Castells, David Harvey, y Henri Lefebvre, Saskia Sassen o Doreen Massey. Este rastreo panorámico no logra un nivel profundo de análisis, pero permite a la autora hacer un conjunto de conclusiones a modo de premisas preliminares para lo que denomina como Trabajo Social urbano: que la ciudad es una construcción social, que es vivida en forma relacional y compleja, que son altamente diversas y diferenciadas internamente, que reflejan un conjunto de relaciones permeadas por el poder, el privilegio, desigualdad e injusticia, que median las escalas de la política pública, y que su crecimiento no se encuentra desligado de los procesos de la ruralidad. En suma, la autora plantea que "la ciudad, su espacio, lugar y tiempo, es mucho más que un simple telón de fondo para la práctica del Trabajo Social” (Williams, 2016, p. 38).

Esta primera aproximación teórica a la ciudad es profundizada en el capítulo 2, en el que la autora identifica cuatro formas en que ha sido conceptualizada, las que, a su vez, implican diferentes énfasis para el Trabajo Social urbano. En primer lugar, identifica una aproximación a la ciudad como una máquina, es decir, desde perspectivas deterministas, ya sean de índole psico-social, o marxistas -sin embargo, considero que se ubican los enfoques radicales y marxistas dentro de este conjunto de perspectivas deterministas, sin una argumentación acabada-. En segundo lugar, identifica una conceptualización de la ciudad 
Saravia-Cortés

como sistema. Desde esta lógica, se la entiende como un organismo, configurado a partir de un conjunto de subsistemas o subculturas interconectadas. La autora menciona a la Escuela de ecología humana de Chicago como un referente importante en esta línea de pensamiento, mas entiende estas aproximaciones como inherentemente conservadoras, ya que tienden a preferir cambios incrementales en lugar de cambios radicales, en gran medida orientados al déficit y a menudo silenciosos sobre cuestiones de poder. La tercera aproximación identificada concibe la ciudad como una comunidad, o más precisamente, como una comunidad de comunidades. La idea central en este enfoque es que, si los recursos correctos están disponibles para la comunidad, esta podrá administrar sus propias relaciones y responsabilidades, por lo que la labor del Trabajo Social urbano se restringe al empoderamiento de las comunidades para que puedan tomar el control respecto de sus propios destinos y entornos. La autora critica esta aproximación por desconocer los impactos de la estructura social en las comunidades, por ser demasiado localista y fácilmente caer en intentar constantemente inventar la rueda en las distintas comunidades, debido a perspectivas fragmentadas de la ciudad y lo urbano en general. Finalmente, la última aproximación identificada entiende a la ciudad como ambiente. Citando principalmente el trabajo de Zapf (2009), la autora hace referencia al enfoque de persona en ambiente, que desde una perspectiva ecológica entendería el ambiente social asociado a los lugares como las redes familiares, amistad y trabajo. Por otro lado, el concepto de ambiente también sería utilizado según la autora, desde enfoques centrados en la promoción de la sostenibilidad ambiental, concretados principalmente a través del activismo.

Ninguno de estos cuatro enfoques es especialmente defendido por la autora, sino que plantea que en la actualidad se encontraría en desarrollo un nuevo paradigma para la acción del Trabajo Social urbano, que ella denomina como un modelo de diseño cooperativo de la ciudad. Ello implicaría cambios en cuatro dimensiones centrales: la forma en que se comprenden el espacio y el lugar en las dinámicas urbanas, la forma en que se entienden los asuntos relacionados con las escalas de intervención, las aproximaciones al concepto de sustentabilidad social, y la relación con expresiones de las nuevas formas de gobernanza de los asuntos públicos.

Teniendo lo anterior en consideración, en el capítulo 3 la autora analiza cómo un conjunto de trabajadores sociales en ejercicio en Australia, conciben la ciudad y las implicancias de un enfoque de Trabajo Social urbano. En términos generales, los profesionales pueden reflexionar de forma más o menos informada en torno a la ciudad y sus dinámicas, pero no respecto de cómo ello se vincularía con el quehacer del Trabajo Social propiamente tal. Luego de un análisis pormenorizado de entrevistas y grupos de discusión a dichos profesionales, la autora propone un conjunto de requerimientos para el Trabajo Social urbano, algunos de los cuales son: la consideración critica de las dinámicas urbanas, sus impactos, y el rol del trabajador social en la configuración de la ciudad; un entendimiento actualizado del funcionamiento del Estado a nivel local, así como de las 
Saravia-Cortés

oportunidades y constricciones que implica las formas de gobernanza neoliberal imperantes; la creación y coordinación de foros, plataformas y comunidades profesionales que permitan generar alianzas y transferir conocimiento; un entendimiento acabado de la multi escalaridad de los fenómenos socioespaciales que permita diseñar intervenciones sociales que vayan más allá de los limites político-administrativos del Estado; el impulso de prácticas profesionales creativas que impliquen modelos participativos transparentes; el compromiso con nuevas formas de resistencia y movilizaciones sociales; entre otros.

En el capítulo 4 la autora se aboca a delinear los principales desafíos investigativos que implica una perspectiva urbana en Trabajo Social. En primer lugar, muestra que la profesión cuenta con una tradición investigativa históricamente vinculada a lo urbano, dando entre otros, el ejemplo de la influencia de Charles Booth en la escuela de Chicago, a través de su trabajo pionero de mapeo de la ciudad de Londres. Indica también lo amplio del campo de estudios que se abre al asumir una perspectiva urbana en Trabajo Social, ya que como plantea, los lugares se expresan en diferentes escalas -el hogar, el jardín, el barrio, el pueblo o la ciudad, la región, el país en su conjunto-, cada uno de los cuales da lugar a diversos fenómenos socioespaciales susceptibles de ser investigados. En esta tarea, propone, Trabajo Social debiera entablar diálogos interdisciplinarios con diversas disciplinas vinculadas a lo urbano, y acercarse al uso de metodologías y técnicas de investigación propias de dichas disciplinas, como los sistemas de información geográfica, así como enfoques etnográficos, y enfoques que enfatizan el análisis de la movilidad espacial cotidiana, entre otros. Para ello, plantea que es necesario el desarrollo de asociaciones colaborativas entre la academia y las comunidades, y entre las agencias que llevan a cabo las intervenciones y sus respectivos usuarios. Es decir, se aboga por modelos de investigación que funcionen con una lógica de co-producción del conocimiento científico que retroalimente al Trabajo Social urbano.

Como se aprecia, Williams (2016) no solo fundamenta la relevancia de una perspectiva urbana para el Trabajo Social contemporáneo, sino que también identifica un conjunto de desafíos para el quehacer de los profesionales en ejercicio, y para el quehacer investigativo de los académicos en escuelas de Trabajo Social. A ello se adiciona un desafío relacionado con la formación en Trabajo Social, que es desarrollado en el capítulo final del libro por Susie Costello y Julian Raxworthy. Los autores dan cuenta de una experiencia formativa realizada en la School of Global Urban and Social Studies de la RMIT University, en Melbourne, Australia. Esta consistió en la inserción de estudiantes de Trabajo Social y arquitectura en una experiencia de intervención urbana desde una agencia del gobierno local en el contexto de un barrio vulnerable. Se destacan varios aspectos positivos de esta, pero también se identifican un conjunto de aspectos a tener en consideración para el desarrollo exitoso de iniciativas de esta naturaleza, tales como la necesidad de desarrollar diálogos interdisciplinares que permitan el esclarecimiento de las jergas profesionales para que tanto académicos como estudiantes de las distintas disciplinas implicadas puedan comprenderse 
Saravia-Cortés

mutuamente. Se destaca también la necesidad de establecer metas claras y transparentes para todos los implicados en el proceso de interrelación.

En suma, el trabajo de Williams (2016) hace un aporte importante para una mejor comprensión del Trabajo Social en las ciudades. Sin embargo, estimo que es necesario avanzar hacia un análisis más pormenorizado respecto de las implicancias precisas de diferentes opciones teóricas, no solo para fines investigativos o meramente académicos, sino también en el diseño de intervenciones sociales urbanas concretas. Ello implicaría avanzar también hacia análisis que permitan desentrañar los fundamentos teóricos -probablemente implícitos- de las intervenciones urbanas, como lo hace el interesante trabajo realizado por Tapia-Barría (2018) en el contexto chileno, aunque sin un vínculo directo con el Trabajo Social. Por otro lado, estimo que la lectura que Williams (2016) realiza de las posibilidades de análisis urbano desde perspectivas marxistas debe ser profundizada. De hecho, trabajos como el de Abreu (2018) o Saravia (2019), surgidos desde el contexto latinoamericano, muestran que perspectivas marxistas resultan ser útiles para el análisis de las realidades urbanas y de las intervenciones de estas.

Con todo, el aporte de Williams (2016) nutre un subcampo del Trabajo Social que, al parecer, se encuentra desde no hace mucho tomando una fuerza incipiente a nivel internacional, y en el que diálogo entre distintas perspectivas teóricas y distintos territorios resultará de vital importancia. Desde América Latina podemos y debemos entrar en esta perentoria discusión.

\section{Referencias bibliográficas}

Abreu, M. H. (2018). Fronteiras, armadilhas e muros: contribuições teórico-metodológicas para o debate sobre território. Revista Katálysis, 21(2), 261-270. Recuperado de https:/ / periodicos.ufsc.br/index.php/katalysis/article/view/1982$02592018 v 21 n 2$ p261.

Addams, J. (2013). Hull House: el valor de un centro social. Madrid, España: Colegio general del Trabajo Social.

Orellana, V. y Panez, A. (2016). El debate sobre la cuestión urbana y el Trabajo Social: contribuciones críticas para pensar la profesión en territorios de relegación urbana. Perspectivas, 27, 59-88. doi: 10.29344/07171714.27.411

Saravia, F. (2019). Espacio e intervención en trabajo social a partir de Lefebvre. Cinta de Moebio, (66), 281-294. doi: 10.4067/S0717-554X2019000300281.

Saravia, F., Urquieta, M.A., y Ortega, M.B. (2020). Espacialidades en la intervención social: debates para América Latina. Santiago de Chile: RIL editores. 
Saravia-Cortés

Tapia-Barría, V. (2018). Geografías de la contención: el rol de las políticas de escala barrial en el Chile neoliberal. Scripta Nova. Revista Electrónica de Geografía y Ciencias Sociales, 22(592), 1138-9788. doi: 10.1344/sn2018.22.20272

Williams, Ch. (Ed.). (2016). Social Work and the City: Urban Themes in 21st-Century Social Work. Melbourne, Australia: Palgrave Macmillan.

Wohl, A. (1971). Octavia Hill and The Homes of the London Poor. TheJournal of British Studies, 10(2), 105-131.

Zapf, M. (2009). Social Work and the Environment: understanding People and Place. Toronto, Canadá: Canadian Scholars' Press Inc. 


\section{OTROS ARTÍCULOS DE PROSPECTIVA No. 31 DE 2021}

\section{PRESENTACIÓN}

Presentación. Reflexiones sobre desafios al publicar sistematizaciones

Rosa María Cifuentes-Gil

\section{EDITORIAL}

Reflexiones sobre Trabajo Social: aportes de la Sistematización

María Rocío Cifuentes-Patiño

\section{ARTÍCULOS}

Hacer lo que se sabe, pensar lo que se hace. La sistematización como modalidad investigativa Alfonso Torres-Carrillo

Aportes y desafios de la Sistematización de experiencias en el Trabajo Social y la extensión crítica. Apuntes y reflexiones desde la perspectiva de la Educación Popular

María Rosa Goldar

Valeria Chiavetta

La sistematización en Trabajo Social y la epistemología feminista del punto de vista. Diálogos sobre la producción de conocimiento sustentada en experiencias

Ruth Noemí Parola

María Florencia Linardelli

La Sistematización investigativa de las experiencias: del baile de los que sobran a la fiesta de los que faltan

María Belén Ortega-Senet

Sistematización y Trabajo Social en Chile. El largo y sinuoso camino

Patricia Lorena Castañeda-Meneses

Ana María Salamé-Coulon
Sentipensar la pandemia COVID-19 desde la sistematización de la experiencia en Trabajo Social: reflexiones del profesor Oscar Jara Holliday

Elia Sepúlveda-Hernández

La sistematización de experiencias, una investigación social cualitativa que potencia buenas prácticas de convivencia y gobierno. La experiencia de un conjunto residencial multifamiliar en Cali, Colombia Martha Lucia Echeverry-Velásquez Manuela Prada-Dávila

Construcción de subjetividades epistemológicaspolíticas de profesoras y profesores de Investigación social en una universidad privada y confesional en Bogotá

Giovanni Mora-Lemus

Sistematización de la experiencia Reconocimiento de los derechos humanos del adulto mayor en dos familias residentes en Cali y Valledupar (Colombia)

Lina María Cuello-Lacouture

Jimena del Pilar Jaramillo-Jaramillo

La memoria transformadora como estrategia de intervención profesional en los procesos de reconciliación social: comprensión a partir de mujeres campesinas, excombatientes y jóvenes en Manizales, Colombia

Yeimmy Stephania Corredor-Sotelo

Juliana Fuertes-Fuertes

Sistematización de una estrategia de educación informal implementada en personas privadas de la libertad en el establecimiento penitenciario de mediana seguridad y carcelario de Barranquilla, Colombia

Rafael Humberto Herrera-Mercado Rafael Alberto Zambrano-Vanegas 
Aportes significativos del proceso de intervención comunitaria con la Escuela Popular de Comunicación Alternativa Jaime Garzón de la ciudad de Cúcuta, Colombia

Carlos Lasso-Urbano

La sistematización de la intervención como metodología de investigación en Trabajo Social. Importancia práctica y teórica de la fase de recogida de datos en la intervención social según experiencia del Programa de Apoyo a las Familias en Zaragoza, España

Elisa Esteban-Carbonell

Nuria Del Olmo-Vicén

Papel de la sistematización de experiencias en los procesos de evaluación de intervenciones de salud pública en la Comuna Saludable por la Paz, Cali - Colombia

Jenny Faisury Peña-Varón

Paola Andrea Marín-Velásquez

Janeth Mosquera-Becerra

Experiencia de intervención social en hogares comunitarios integrales del barrio Alfonso Bonilla Aragón, Cali - Colombia

Julián Alexander Montaño-Cárdenas

Las políticas sociales y el gobierno de la "población indígena". Estrategias y regulaciones en el multiculturalismo chileno

Rodrigo Agustín Navarrete-Saavedra

Representaciones sociales sobre estilo de autoridad y tipos de interacción en cuidadores de residencias de protección infantil en Chile

Marcelo Gallegos-Fuentes
Carmen Gloria Jarpa-Arriagada

Reflexiones sobre inseguridad social y cuestiones penales. Una respuesta estratégica a partir de experiencias de cooperativismo con ex detenidos en Argentina

Analia Elizabeth Otero

Yael Yanina Barrera

Desarrollo y salud: la emergencia de un nuevo paradigma

Jesús María Sánchez-Ordóñez

Trabajo Social en ejercicio libre: la perspectiva profesional en España

Paula Frieiro-Padín

Tamara Fernández-Arias

Rubén González-Rodríguez

\section{RESEÑAS}

Social Work and the City: Urban Themes in 21stCentury Social Work

Felipe Saravia-Cortés

Respuestas del Trabajo Social ante emergencias sociales y problemáticas sociales complejas de México y España

Felipe Saravia-Cortés

El feminismo, el género y la profesionalización del trabajo social en Colombia (1936-2004)

Ambar Oriana Serna-Lombo

El puño invisible. Arte, revolución y un siglo de cambios culturales

Carlos Arturo Robledo-Marín

\section{PROSPECTIVA}

No. $31 \bullet$ ene.-jun. 2021

e-ISSN: 2389-993X • Universidad del Valle 